\title{
NOTE ON DEGREE OF APPROXIMATION BY BOUNDED ANALYTIC FUNCTIONS: PROBLEM $\beta\left({ }^{1}\right)$
}

\author{
BY \\ J. L. WALSH
}

In the theory of approximation by bounded analytic functions of a complex variable, Problem $\alpha$ is the study of the relation between degree of approximation on a point set $E$ to continuity properties on $E$ of the function approximated; Problem $\beta$ is the study of the relation between degree of approximation on $E$ to continuity properties on a different point set $E^{\prime}$ of the function $f(z)$ approximated; an example of Problem $\beta$ is the study of degree of approximation on the unit disc $E:|z| \leqq 1$ as related to continuity properties of $f(z)$ on the circumference $E^{\prime}:|z|=R(>1)$, where $f(z)$ is analytic throughout $|z|<R$. The present writer has recently published [1] a general result (Theorem 5 below) on Problem $\beta$, and now wishes to indicate a stronger result (Theorem 6 below). The older result deals with Lipschitz conditions in the hypothesis, while the new one deals with integrated Lipschitz conditions; the latter were introduced into the theory of degree of trigonometric approximation in theorems stated without proof by Hardy and Littlewood [2] and, with additions, proved in detail by Quade [3]. The present note is made possible by an investigation on integrated Lipschitz conditions for the complex variable made by Walsh and Russell [4]. The note is divided into two parts, devoted respectively to the study of classes of functions involving integrated Lipschitz conditions and to applications to approximation by bounded analytic functions.

Part I. Classes $H(k, \alpha, p)$ and $H^{\prime}(k, \alpha, p)$. If $\gamma$ is the unit circumference $|z|=1$, if the function $f(z)$ is analytic in the unit disc $|z|<1$, and if the means

$$
M_{p}\left[f\left(r e^{i \theta}\right)\right]=\left\{\int_{0}^{2 \pi}\left|f\left(r e^{i \theta}\right)\right|^{p} d \theta\right\}^{1 / p}, \quad 1 \leqq p,
$$

are bounded for $r<1$, the function is said to be of class $H_{p}$ on $\gamma$. It is customary to use the terminology on $\gamma$ even though the properties of $f(z)$ required refer to the neighborhood of $\gamma$. A function $f(z)$ analytic in the interior or exterior (including the point at infinity) of an analytic Jordan curve $J$ is said to be of class $H_{p}$ on $J$ if the transform of $f(z)$ is of class $H_{p}$ on $\gamma$ when the interior or exterior of $J$ is mapped one-to-one and conformally onto the interior of $\gamma$. This property is invariant under conformal mapping of the

Received by the editors October 19, 1959.

(1) This research was sponsored (in part) by the U. S. Air Force, Air Research and Development Command. 
interior or exterior of $J$ onto the interior or exterior of an analytic Jordan curve. If $f(z)$ is of class $H_{p}$ on $\gamma$ [or $J$ ], boundary values for approach "in angle" exist almost everywhere on $\gamma$ [or $J$ ], and $f(z)$ is of class $L_{p}$ there. A Cauchy integral for $f(z)$ may be taken over $\gamma$ [or $J$ ].

If $k$ is a non-negative integer, the function $f(z)$ is said to be of class $H(k, \alpha, p), 0<\alpha<1, p \geqq 1$, on the unit circle $\gamma$ if it is of class $H_{p}$ there, and if the $k$ th derivative $f^{(k)}(z) \equiv F(s)$ of the boundary values on $\gamma$ exists and satisfies

$$
\int_{\gamma}|F(s+h)-F(s)|^{p} d s \leqq A|h|^{\alpha p},
$$

$s$ being arc length on $\gamma$; here and below $A$ with or without subscript indicates a constant independent of $h, n, z, w$, whose meaning may change from one formula to another. A function $f(z)$ is of class $H(k, \alpha, p)$ on $\gamma$ where $k$ is a negative integer, $0<\alpha<1$, if $f\left(r e^{i \theta}\right)$ is analytic for $r<1$ with

$$
M_{p}\left[f\left(r e^{i \theta}\right)\right] \leqq A(1-r)^{k+\alpha} .
$$

A necessary and sufficient condition that a function $f(z)$ analytic for $r<1$ belong to $H(0, \alpha, p)$ on $\gamma, 0<\alpha<1$, is $M_{p}\left[f^{\prime}\left(r e^{i \theta}\right)\right] \leqq A_{1}(1-r)^{\alpha-1}$; if $M_{p}\left[f\left(r e^{i \theta}\right)\right]$ $\leqq A(1-r)^{\mu}, \mu<0$, then $M_{p}\left[f^{\prime}\left(r e^{i \theta}\right)\right] \leqq A_{1}(1-r)^{\mu-1}$; if $M_{p}\left[f\left(r e^{i \theta}\right)\right] \leqq A(1-r)^{\mu}$, $\mu<-1$, then $M_{p}\left[F\left(r e^{i \theta}\right)\right] \leqq A_{1}(1-r)^{\mu+1}$, where $F(z)$ is any integral of $f(z)$. A function $f(z)$ is said to be of class $H(k, \alpha, p)$ on an analytic Jordan curve $J$, if the transform of $f(z)$ under conformal map of the interior (or exterior) of $J$ onto $|z|<1$ is of class $H(k, \alpha, p)$ on $\gamma$. Thus for each $\alpha(0<\alpha<1)$ and $p(\geqq 1)$ we have on $\gamma$ and on $J$ a sequence of classes $\cdots, H(-2, \alpha, p)$, $H(-1, \alpha, p), H(0, \alpha, p), H(1, \alpha, p), H(2, \alpha, p), \cdots$; the derivative of a function of a class belongs to the next lower class and the indefinite integral belongs to the next higher class. These classes are invariant under conformal mapping of the interior (or exterior) of $J$ onto the interior or exterior of any analytic Jordan curve $[4 ; 5 ; 6]$. There is usually no ambiguity in practice as to whether the interior or exterior is involved, for $f(z)$ is ordinarily known to be analytic on but one side of $J$. A function $f(z)$ is said to be of class $H(k, \alpha, p)$ on a finite number of mutually exterior analytic Jordan curves if it is of that class on each curve. The class is additive with respect to its members.

If $J$ is an analytic Jordan curve, the function $f(z)$ is said to be of class $H^{\prime}(k, \alpha, p)$ on $J$ if $f(z)$ is the sum of a function of class $H(k, \alpha, p)$ on $J$ and a function analytic in a (two-sided) closed neighborhood of $J$. It then follows by Cauchy's integral formula for an annular region containing $J$ that $f(z)$ is the sum of a function of class $H(k, \alpha, p)$ on $J$ and a function analytic throughout the closed exterior (interior) of $J$. The class $H^{\prime}(k, \alpha, p)$ on $J$ is clearly invariant under conformal mapping of the interior (exterior) of $J$ onto the unit disc; indeed, condition $(2)$ is invariant $[4 ; 5 ; 6]$. For each $\alpha(0<\alpha<1)$ and $p(\geqq 1)$ we have on $\gamma$ and on $J$ a sequence of classes $H^{\prime}(k, \alpha, p)$, $k=\cdots,-2,-1,0,1,2, \cdots$; the derivative of each function of a class 
belongs to the next lower class; the integral belongs to the next higher class if single-valued, and if not single-valued differs from a function of the next higher class by a function analytic but multiple-valued on $\gamma$ or $J$.

The limiting case $(p \rightarrow \infty)$ of the class $H^{\prime}(k, \alpha, p), k \geqq 0,0<\alpha<1$, is the class $H^{\prime}(k, \alpha, \infty)$ of functions $f(z)$ each analytic in a one-sided neighborhood of the analytic Jordan curve $J$, continuous on $J$, whose $k$ th derivative $F(s)$ satisfies there a Lipschitz condition $|F(s+h)-F(s)| \leqq A|h|^{\alpha}$ with respect to arc length $s$ on $J$ instead of (2). This is primarily a local condition, and these classes are more easily managed than the classes $H^{\prime}(k, \alpha, p)$. The class $H^{\prime}(k, \alpha, \infty)$ on $\gamma:|z|=1$ for $k<0$ requires analyticity in an annulus $(0<) \rho$ $<|z|<1$, together with the inequality $\left|f\left(r e^{i \theta}\right)\right| \leqq A_{1}(1-r)^{k+\alpha}, r<1$. The class $H^{\prime}(k, \alpha, \infty)$ for $k<0$ on an analytic Jordan curve $J$ is defined as the set of transforms of the functions of $H^{\prime}(k, \alpha, \infty)$ on $\gamma$ when the interior (exterior) of $J$ is mapped onto the interior of $\gamma$. As with $p<\infty$, there is for each $\alpha$, $0<\alpha<1$, a sequence of classes $H^{\prime}(k, \alpha, \infty)$ on $J, k=\cdots,-2,-1,0,1$, $2, \ldots$; the derivative of a function of a class belongs to the next lower class and (with qualifications as before) the integral to the next higher class. These classes are invariant under conformal mapping of the interior (or exterior) of $J$ onto the interior (or exterior) of any analytic Jordan curve.

For later use we need to consider some detailed properties of the classes already defined. The class $H(k, \alpha, p), k \geqq 0$, is completely characterized by degree of approximation [4]:

THEOREM 1. If $J$ is an analytic Jordan curve and the function $f(z)$ is defined almost everywhere on $J$, a necessary and sufficient condition that $f(z)$ be the set of boundary values of a function of class $H(k, \alpha, p), k \geqq 0,0<\alpha<1, p \geqq 1$, on $J$ is that there exist polynomials $p_{n}(z)$ of respective degrees $n$ in $z$ such that we have $(n=1,2, \cdots)$

$$
\int_{J}\left|f(z)-p_{n}(z)\right|^{p}|d z| \leqq A / n^{(k+\alpha) p} .
$$

The polynomials $p_{n}(z)$ converge uniformly on any closed set interior to $J$, to this function of class $H(k, \alpha, p)$ on $J$.

We shall not prove Theorem 1 in detail here, but mention that thanks to a detailed study of invariance of degree of polynomial approximation under conformal map $[4 ; 5 ; 6]$, it is sufficient to prove Theorem 1 when $J$ is $\gamma:|z|$ $=1$. The first part then follows [4] from the Hardy-Littlewood-Quade theory of trigonometric approximation. As for the second part, by Cauchy's integral formula the sequence $p_{n}(z)$ converges in $|z|<1$ to some function $f_{1}(z)$ analytic in $|z|<1$. A suitably chosen subsequence [4] of the $p_{n}(z)$ converges in the mean on all circles $|z|=r, r \leqq 1$, uniformly with respect to $r$, to the function $f_{1}(z)$ which is therefore of class $H_{p}$. Thus the limit as $r \rightarrow 1$ of the limit in the mean $f_{1}(z)$ of that subsequence on $|z|=r$ is the limit in the mean $f_{1}(z)$ of 
that subsequence on $\gamma$, namely $f(z)$ a.e. on $\gamma$. That $f(z)$ satisfies (2) follows from the trigonometric theory. With $k>0$ it follows also by study [4] of the subsequence that $f^{(k)}(z)$ is of class $H_{p}$ on $\gamma\left({ }^{2}\right)$.

It is now our purpose to extend Theorem 1 to the class $H^{\prime}(k, \alpha, p)$, and thereby to establish useful properties of that class which are invariant under conformal transformation; similar but less refined properties have already been established [4, Theorems 4-8] for the class $H(k, \alpha, p)$.

THEOREM 2. Let $S$ be an annular region bounded by analytic Jordan curves $C_{1}$ and $C_{2}$, with $C_{2}$ interior to $C_{1}$ and 0 interior to $C_{2}$. Let polynomials $p_{n}(z, 1 / z)$ in $z$ and $1 / z$ exist such that we have for a function $f(z)$ analytic on $S+C_{2}$ with boundary values a.e. on $C_{1}(k \geqq 0)$

$$
\int_{C_{j}}\left|f(z)-p_{n}(z, 1 / z)\right|^{p}|d z| \leqq \frac{A}{n^{(k+\alpha) p}}, \quad j=1,2 .
$$

Then $f(z)$ is of class $H^{\prime}(k, \alpha, p)$ on $C_{1}$.

Conversely, if $f(z)$ denotes the set of boundary values almost everywhere on $C_{1}$ of the function $f(z)$ analytic on $S+C_{2}$ and of class $H^{\prime}(k, \alpha, p)$ on $C_{1}$, then there exist polynomials $p_{n}(z, 1 / z)$ of degree $n$ in $z$ and $1 / z$ such that $(4)$ is satisfied.

Let the $p_{n}(z, 1 / z)$ be given. We split $p_{n}(z, 1 / z)$ into polynomials $P_{n}(z)$ and $Q_{n}(1 / z)$ in $z$ and $1 / z$ each of degree $n$, with $Q_{n}(1 / z)=0$ for $z=\infty: p_{n}(z, 1 / z)$ $\equiv P_{n}(z)+Q_{n}(1 / z)$. There is valid the formula

$$
\frac{1}{2 \pi i} \int_{C_{2}} \frac{p_{n}(t, 1 / t) d t}{t-z} \equiv Q_{n}(1 / z), \quad z \text { exterior to } C_{2} .
$$

We may likewise write

$$
\begin{aligned}
\frac{1}{2 \pi i} \int_{C_{1}} \frac{f(t) d t}{t-z} & \equiv f_{1}(z), & & z \text { interior to } C_{1}, \\
\frac{1}{2 \pi i} \int_{C_{2}} \frac{f(t) d t}{t-z} & \equiv f_{2}(z), & & z \text { exterior to } C_{2} ;
\end{aligned}
$$

the functions $f_{1}(z)$ and $f_{2}(z)$ are analytic respectively throughout the interior of $C_{1}$ and exterior of $C_{2}$. Moreover $f_{1}(z)\left[\equiv f(z)-f_{2}(z)\right]$ is analytic in $S$ and has boundary values $f_{1}(z)$ a.e. on $C_{1}$. In particular we have

$$
\int_{C_{2}} \frac{f(t)-p_{n}(t, 1 / t)}{t-z} d t \equiv f_{2}(z)-Q_{n}(1 / z), \quad z \text { on } C_{1},
$$

(2) It is required in [4] in order that $f(z)$ belong to the class $H(k, \alpha, p)$ on $\gamma$ that (among other properties) $f^{(k)}(z)$ shall be of class $H_{p}$. In the present note we have required merely that $f(z)$ shall be of class $H_{p}$, from which the first part of Theorem 1 can be established [4, Theorem D], and the second part of Theorem 1 shows as just outlined that $f^{(k)}(z)$ is of class $H_{p}$ on $\gamma$. 
and by (4) for $j=2$ with use of the Hölder inequality

$$
\mid f_{2}(z)-Q_{n}(1 / z) ! \leqq \frac{A_{1}}{n^{k+\alpha}}, \quad z \text { on } C_{1} .
$$

From (4) for $j=1$ and (5) we now write

$$
\int_{C_{1}}\left|f(z)-f_{2}(z)-P_{n}(z)\right| p|d z| \leqq \frac{A_{2}}{n^{(k+\alpha) p}},
$$

so by Theorem 1 the function $f_{1}(z)$ is of class $H(k, \alpha, p)$ on $C_{1}$ and $f(z)$ is of class $H^{\prime}(k, \alpha, p)$ on $C_{1}$.

To prove the second part of Theorem 2, we note that by hypothesis we have $f(z) \equiv f_{1}(z)+f_{2}(z)$ on $S+C_{1}+C_{2}$, where $f_{1}(z)$ is of class $H(k, \alpha, p)$ on $C_{1}$, hence analytic throughout the interior of $C_{1}$, and $f_{2}(z)$ is analytic throughout the closed exterior of $C_{2}$. By Theorem 1 , there exist polynomials $P_{n}(z)$ in $z$ of respective degrees $n$ satisfying

$$
\int_{C_{1}}\left|f_{1}(z)-P_{n}(z)\right| p|d z| \leqq A / n^{(k+\alpha) p} .
$$

Use of Cauchy's integral formula over $C_{1}$ now yields

$$
\left|f_{1}(z)-P_{n}(z)\right| \leqq A_{1} / n^{k+\alpha},
$$
$z$ on $C_{2}$.

From the analyticity of $f_{2}(z)$ on and exterior to $C_{2}$, since after a transformation $z^{\prime}=1 / z$ the transform of $f_{2}(z)$ is of class $H(k, \alpha, p)$ on the image of $C_{2}$, it follows that there exist polynomials $Q_{n}(1 / z)$ in $1 / z$ of respective degrees $n$ satisfying

$$
\begin{aligned}
\int_{C_{2}}\left|f_{2}(z)-Q_{n}(1 / z)\right| p|d z| & \leqq A_{2} / n^{(k+\alpha) p}, \\
\left|f_{2}(z)-Q_{n}(1 / z)\right| & \leqq A_{3} / n^{k+\alpha}, \quad z \text { on } C_{1} .
\end{aligned}
$$

We may now obtain inequalities (4) with $p_{n}(z, 1 / z) \equiv P_{n}(z)+Q_{n}(1 / z)$. Theorem 2 is established.

Closely related to Theorem 2 is

THEOREM 3. Let $S_{1}$ be an annular region containing in its interior the annular region $S$ bounded by analytic Jordan curves $C_{1}$ and $C_{2}$, with $C_{2}$ interior to $C_{1}$. Let $f(z)$ be analytic on $S+C_{2}$ with boundary values a.e. on $C_{1}$, and let the functions $f_{n}(z)$ be analytic in $S_{1}$ and satisfy $(k \geqq 0)$

$$
\begin{aligned}
\int_{C_{j}}\left|f(z)-f_{n}(z)\right| p|d z| & \leqq \frac{A}{n^{(k+\alpha) p}}, & j=1,2, \\
\left|f_{n}(z)\right| \leqq A R^{n}, & & z \text { in } S_{1} .
\end{aligned}
$$

Then $f(z)$ is of class $H^{\prime}(k, \alpha, p)$ on $C_{1}$. 
Let $\Gamma_{1}$ and $\Gamma_{2}$ interior to $\Gamma_{1}$ be analytic Jordan curves in $S_{1}$ bounding an annular region $S_{2}$ containing $S$, and let $\Gamma_{1}^{\prime}$ and $\Gamma_{2}^{\prime}$ interior to $\Gamma_{1}^{\prime}$ be analytic Jordan curves in $S_{2}$ bounding an annular region containing $S$. The functions $f_{n 1}(z)$ and $f_{n 2}(z)$ defined by the equations

$$
\begin{array}{rlrl}
f_{n 1}(z) & \equiv \frac{1}{2 \pi i} \int_{\Gamma_{1}} \frac{f_{n}(t) d t}{t-z}, & & z \text { interior to } \Gamma_{1}, \\
f_{n 2}(z) \equiv \frac{1}{2 \pi i} \int_{\Gamma_{2}} \frac{f_{n}(t) d t}{t-z}, & & z \text { exterior to } \Gamma_{2},
\end{array}
$$

are analytic respectively throughout the interior of $\Gamma_{1}$ and exterior of $\Gamma_{2}$; in $S_{2}$ we have $f_{n}(z) \equiv f_{n 1}(z)+f_{n 2}(z)$. From (7) follow the inequalities

$$
\begin{array}{ll}
\left|f_{n 1}(z)\right| \leqq A_{1} R^{n}, & z \text { on } \Gamma_{1}^{\prime}, \\
\left|f_{n 2}(z)\right| \leqq A_{1} R^{n}, & z \text { on } \Gamma_{2}^{\prime}
\end{array}
$$

Choose the origin 0 interior to $C_{2}$. For a suitably chosen $\rho(>1)$ there exist $[10, \S 4.5]$ polynomials $P_{n k}(z)$ and $Q_{n k}(1 / z)$ respectively of degree $k$ in $z$ and $1 / z$ such that

$$
\begin{aligned}
\left|f_{n 1}(z)-P_{n k}(z)\right| & \leqq A_{2} R^{n} / \rho^{k}, & & z \text { on } C_{1}, \\
\left|f_{n 2}(z)-Q_{n k}(1 / z)\right| & \leqq A_{2} R^{n} / \rho^{k}, & & z \text { on } C_{2} .
\end{aligned}
$$

If the integer $\lambda$ is chosen so that $\rho^{\lambda}>R$, we have consequently

$$
\left|f_{n}(z)-p_{n, \lambda n}(z, 1 / z)\right| \leqq 2 A_{2}\left(R / \rho^{\lambda}\right)^{n}, \quad z \text { on } C_{1}+C_{2},
$$

with $p_{n k}(z, 1 / z) \equiv P_{n k}(z)+Q_{n k}(1 / z)$. From (6) and (9) we may write

$$
\int_{C_{j}}\left|f(z)-p_{n, \lambda n}(z, 1 / z)\right|^{p}|d z| \leqq \frac{A_{3}}{n^{(k+\alpha) p}}, \quad j=1,2 .
$$

The polynomials $p_{n, \lambda n}(z, 1 / z)$ are here defined merely for the degrees $\lambda n$ $=\lambda, 2 \lambda, 3 \lambda, \cdots$, but if we set

$$
\begin{array}{lrl}
p_{j}(z, 1 / z) & \equiv p_{n, \lambda n}(z, 1 / z), & \lambda n \leqq j<\lambda(n+1), \\
p_{j}(z, 1 / z) \equiv 0, & 1 \leqq j<\lambda,
\end{array}
$$

the $p_{j}(z, 1 / z)$ are defined and of degree $j$ for every $j(>0)$, and we have the equivalent of (4). Theorem 3 follows from the first part of Theorem $2\left(^{3}\right)$.

It is of considerable interest that the first part of Theorem 2 also follows from Theorem 3. In fact, from (4) we obtain

(3) It may be noticed that Theorems 2 and 3 admit an extension, in which the second members of (4) and (6) are replaced by suitably chosen $\epsilon_{n}^{p}$, and the conclusion refers to a class of functions $f_{1}(z)+f_{2}(z)$, where $f_{1}(z)$ is characterized by $p$ th power approximation of order $\epsilon_{n}$ on $C_{1}$ by polynomials $p_{n}(z)$ in $z$ of respective degrees $n$, and the $f_{2}(z)$ are arbitrary functions analytic on $C_{1}$. Compare $[5 ; 6]$. 


$$
\int_{C_{j}}\left|p_{n}(z, 1 / z)\right| p|d z| \leqq A_{4},
$$

whence $[11$, Lemma $]$ we have the equivalent of $(7)$

$$
\left|p_{n}(z, 1 / z)\right| \leqq A_{5} R^{n}, \quad R>1
$$

in the annular region bounded by the level locus $g(z)=\log R$ of Green's function $g(z)$ for the exterior of $C_{1}$ with pole at infinity and by the level locus $g_{0}(z)=\log R$ of Green's function $g_{0}(z)$ for the interior of $C_{2}$ with pole at 0 . The hypothesis of Theorem 3 is satisfied and the first part of Theorem 2 follows.

The content of Theorem 3 is clearly unchanged if $S_{1}$ is transformed by a one-to-one conformal transformation which does not change the topological requirements. If $S$ is an arbitrary annular region whose closure lies in the closure of an annular region $S_{1}$, with the geometric notation of Theorem 3 , and if $f(z)$ is an arbitrary function of class $H^{\prime}(k, \alpha, p)$ on $C_{1}$, analytic on $S+C_{2}$, the analytic functions $f_{n}(z)$ exist satisfying (6) and (7); indeed, the second part of Theorem 2 yields (4), and (10) in $S_{1}$ with suitably chosen 0 . Consequently we have for $k \geqq 0$ :

ThEOREM 4. If $f(z)$ is of class $H^{\prime}(k, \alpha, p)$ on the analytic Jordan curve $C_{1}$, and if $C_{1}$ is transformed by a one-to-one conformal transformation onto a curve $C_{1}^{\prime}$, then the transform of $f(z)$ is of class $H^{\prime}(k, \alpha, p)$ on $C_{1}^{\prime}$.

Of course if $C_{1}$ is so mapped onto $C_{1}^{\prime}$, a neighborhood of $C_{1}$ is correspondingly mapped onto a neighborhood of $C_{1}^{\prime}$ [4, Lemma 2].

Theorem 4, involving the invariance of the class $H^{\prime}(k, \alpha, p)$ under conformal transformation of $C_{1}$, is clearly much stronger than the previous remark concerning the invariance of the class $H(k, \alpha, p)$ on an analytic Jordan curve $J$ under conformal map of the interior of $J$ onto the interior of another analytic Jordan curve.

It is part of the significance of Theorem 4 that if $f(z)$ is of class $H^{\prime}(k, \alpha, p)$ on an analytic Jordan curve $C_{1}$, and if $S$ is an annular region bounded by $C_{1}$ and an analytic Jordan curve $C_{2}$ interior to $C_{1}$ such that $f(z)$ is analytic on $S+C_{2}$, then on the level loci of the function $U(z)$ harmonic in $S$, continuous in the closed region and equal to unity and zero on $C_{1}$ and $C_{2}$, the norm (i.e., the $p$ th power mean) of $f(z)$ is uniformly bounded. Indeed, it follows from the theorem [9] on conformal mapping used below, here with $\mu=\nu=1$ (and indeed is well known) that $S$ can be mapped onto an annular region $S^{\prime}: 0<\rho_{1}$ $<|w|<1$; the level loci in $S^{\prime}$ are the circles $|w|=$ const, and the classical properties of the class $H_{p}$ lead to the result. It is a matter of indifference whether the norm is measured by integration before or after this mapping of $S$ onto $S^{\prime}$.

It remains to establish Theorem 4 when $k<0$; we give the details for 
$k=-1$. With the geometric notation of Theorem 3 , let $f(z)$ be of class $H^{\prime}(-1, \alpha, p)$ on $C_{1}$; it is sufficient to show that $f(z)$ remains of class $H^{\prime}(-1, \alpha, p)$ when $S$ is mapped onto an annulus $S^{\prime}: 0<\rho_{1}<|w|<1$ by a transformation $w=\phi(z)$ analytic in the closure of $S$. We assume, as we may with no loss of generality, that the integral $F(z)$ of $f(z)$ is single-valued in $S$, a property which may be assured by adding to $f(z)$ a suitable constant multiple of $1 /\left(z-z_{0}\right)$, where $z_{0}$ lies interior to $C_{2}$; this addition does not alter the fact that $f(z)$ is of class $H^{\prime}(-1, \alpha, p)$. The function $F(z)$ is of class $H^{\prime}(0, \alpha, p)$ on $C_{1}$, so by Theorem 4 with $k=0$ the function $F_{1}(w) \equiv F(z)$ is of class $H^{\prime}(0, \alpha, p)$ on $\gamma:|w|=1$. Then (compare (3)) $F_{1}^{\prime}(w) \equiv d\left[F_{1}(w)\right] / d w$ is and hence $d[F(z)] / d z \equiv F_{1}^{\prime}(w) \cdot \phi^{\prime}(z)$ is of class $H^{\prime}(-1, \alpha, p)$ on $\gamma$, which we set out to prove. Theorem 4 can be proved also when $k<-1$ by precisely this same method, namely relying on the part of Theorem 4 already proved and the definition (3). Thanks to Theorem 4, the property expressed by (3), like the boundedness of the means, may be used not merely with reference to $S^{\prime}$ but with reference to the level loci in any annular region bounded in part by $C_{1}$ within which $f(z)$ is analytic. This remark is of fundamental importance in the sequel; its proof is one of the primary objects of Part I.

Part II. Approximation by bounded analytic functions. Theorems 1-4 are concerned with Problem $\alpha$ in the theory of approximation. We are now in a position to consider the principal topic that concerns us, namely Problem $\beta$. The main theorem of [1] is primarily devoted to the following theorem and its converse:

THEOREM 5. Let $D$ be a finite region of the z-plane whose boundary consists of mutually disjoint Jordan curves $B_{1}, B_{2}, \cdots, B_{\mu}, C_{1}, C_{2}, \cdots, C_{\nu}$, and let $U(z)$ be the function harmonic in $D$, continuous in the closure of $D$, equal to zero and unity on $B=\sum B_{i}$ and $C=\sum C_{j}$ respectively. For every $\sigma, 0<\sigma<1$, let $\Gamma_{\sigma}$ denote the locus $U(z)=\sigma$ in $D$, and let $D_{\sigma}$ denote the subset $0<U(z)<\sigma$ of $D$, whose boundary is $B+\Gamma_{\sigma}$.

If $\Gamma_{\rho}$ has no multiple point, and if the function $f(z)$ is analytic in $D_{\rho}$, continuous on $B$, and of class $H^{\prime}(k, \alpha, \infty)$ on $\Gamma_{\rho}, 0<\alpha<1$, then there exist functions $f_{n}(z)$ analytic in $D$ and continuous on $B$ such that $(n=1,2,3, \cdots)$

$$
\begin{aligned}
\left|f(z)-f_{n}(z)\right| & \leqq A_{1} e^{-n \rho / \tau} / n^{k+\alpha}, & & \text { zon } B, \\
\left|f_{n}(z)\right| & \leqq A_{2} e^{(1-\rho) / \tau} / n^{k+\alpha}, & & z \text { in } D,
\end{aligned}
$$

where $2 \pi \tau$ is the total variation along $\Gamma_{\rho}$ of the function conjugate to $U(z)$.

The main result of the present note is to weaken the hypothesis of Theorem 5 without changing the conclusion:

Theorem 6. Theorem 5 remains valid if in the hypothesis on $f(z)$ the class $H^{\prime}(k, \alpha, \infty)$ is replaced by $H^{\prime}(k, \alpha, p), p \geqq 1$.

The region $D$ can be mapped [9] onto a region $D^{\prime}: e^{d_{0}}<|u(z)|<e^{d_{1}}$, 


$$
\begin{aligned}
u(z) \equiv \frac{\left(z-a_{1}\right)^{m_{1}}\left(z-a_{2}\right)^{m_{2}} \cdots\left(z-a_{\mu}\right)^{m_{\mu}}}{\left(z-b_{1}\right)^{n_{1}}\left(z-b_{2}\right)^{n_{2}} \cdots\left(z-b_{v}\right)^{n}}, \\
m_{j}>0, n_{j}>0, \sum m_{i}=\sum n_{i}=1,
\end{aligned}
$$

where the $a_{j}$ and $b_{j}$ are finite and are separated from $D^{\prime}$ by the images of the $B_{j}$ and $C_{j}$ respectively. It is no loss of generality to prove Theorem 6 , as we shall do, for the case that $D$ is replaced by $D^{\prime}$. We suppose $D\left(=D^{\prime}\right)$ to lie interior to $C_{1}$, and we use a series of interpolation designed [7] specifically to apply to this specialized region $D$, namely for a function $f_{1}(z)$ analytic in each point $a_{i}$ the series

$$
f_{1}(z) \equiv \sum_{0}^{\infty} c_{n} u_{n}(z), \quad u_{0}(z) \equiv 1, \quad u_{n}(z) \equiv \frac{\left(z-\alpha_{1}\right) \cdots\left(z-\alpha_{n}\right)}{\left(z-\beta_{1}\right) \cdots\left(z-\beta_{n}\right)}
$$

where each $\alpha_{j}$ is some $a_{i}$ and each $\beta_{j}$ is some $b_{i}$, and we have

$$
0<A_{3} \leqq\left|\frac{u_{n}(z)}{[u(z)]^{n}}\right| \leqq A_{4},
$$

uniformly for all $n$ and for all $z$ on any compact set containing no $a_{j}$ or $b_{j}$. If $N_{n j}$ denotes the number of times $b_{j}$ occurs in the set $\beta_{1}, \beta_{2}, \cdots, \beta_{n}$, the number $N_{n j}$ is monotonic nondecreasing as $n$ increases, and $n / N_{n j}$ is bounded for all $j$ and $n \geqq \nu$.

For all $z$ in $D_{\rho}$, the function $f(z)$ can be represented

$$
\begin{gathered}
f(z) \equiv \phi_{1}(z)+\phi_{2}(z), \quad \phi_{1}(z) \equiv \sum \phi_{j 1}(z), \quad \phi_{2}(z) \equiv \sum \phi_{j 2}(z), \\
\phi_{j 1}(z) \equiv \frac{1}{2 \pi i} \int_{\gamma_{j}} \frac{f(t) d t}{t-z}, \quad \phi_{j 2}(z) \equiv \frac{1}{2 \pi i} \int_{B_{j}} \frac{f(t) d t}{t-z},
\end{gathered}
$$

where $\gamma_{j}$ is a suitably chosen rectifiable Jordan curve in $D_{\rho}$ near $\Gamma_{\rho}$, where $\gamma_{j}$ depends on $z$ and there is precisely one $\gamma_{j}$ near each component of $\Gamma_{\rho}$, where $z$ lies in a region bounded by a (not necessarily proper) subset of the $B_{j}$ and a subset of the $\gamma_{j}$, and where the integrals are taken in the positive sense with respect to the open subset of $D_{\rho}$ bounded by $B$ and the $\gamma_{j}$. The function $\phi_{1}(z)$ is analytic throughout $D_{0}^{\prime}:|u(z)|<e^{d_{0}+\rho\left(d_{1}-d_{0}\right)}$, whose boundary is $\Gamma_{\rho}$, and can be represented there [7] by a series (14). Moreover $\phi_{2}(z)$ is analytic on $\Gamma_{\rho}$, so $\phi_{1}(z)$ defined on $\Gamma_{\rho}$ as $f(z)-\phi_{2}(z)$ is of class $H(k, \alpha, p)$ there, by Theorem 4 . It is to be noted that the set $D_{\rho}$ need not be connected, so a particular $\gamma_{j}$ may separate points of $D_{\rho}$; the same notation of $\phi_{j 1}(z)$ then refers for some $z$ in $D_{\rho}$ to a Cauchy integral at points interior to $\gamma_{j}$ and for other $z$ in $D_{\rho}$ to a Cauchy integral at points exterior to $\gamma_{j}$. Also $D_{0}^{\prime}$ need not be connected.

The poles $\beta_{j}$ of $u_{n}(z)$ lie among the $b_{j}$, so lie exterior to $D_{0}^{\prime}$. Suppose now $k \geqq 0$ in Theorem 6 . If $s_{n}(z)$ denotes the sum of the first $n+1$ terms of the series in (14), we have for $z$ in $D_{0}^{\prime}$ the interpolation formula 


$$
\begin{aligned}
\phi_{1}(z)-s_{n}(z) & \equiv \frac{1}{2 \pi i} \int_{\gamma} \frac{\omega_{n}(z) \phi_{1}(t) d t}{\omega_{n}(t)(t-z)}, \\
\omega_{n}(z) & \equiv\left(z-\alpha_{n+1}\right) u_{n}(z) ;
\end{aligned} \quad \gamma=\sum \gamma_{j},
$$

here $s_{n}(z)$ is the unique rational function of degree $n$ whose poles lie in the set $\left(\beta_{1}, \beta_{2}, \cdots, \beta_{n}\right)$ and which interpolates to $\phi_{1}(z)$ in the points $\left(\alpha_{1}, \alpha_{2}, \cdots, \alpha_{n+1}\right)$. A particular case of (17) occurs if $\phi_{1}(z)$ is replaced by an arbitrary rational function $R_{n}(z)$ of degree $n$ all of whose poles lie in the set $\left(\beta_{1}, \beta_{2}, \cdots, \beta_{n}\right)$ :

$$
\begin{aligned}
R_{n}(z) & \equiv \frac{c_{0} z^{n}+c_{1} z^{n-1}+\cdots+c_{n}}{\left(z-\beta_{1}\right) \cdots\left(z-\beta_{n}\right)}, \\
0 & \equiv \frac{1}{2 \pi i} \int_{\gamma} \frac{\omega_{n}(z) R_{n}(t) d t}{\omega_{n}(t)(t-z)},
\end{aligned}
$$

We shall use a combination of (17) and (18):

$$
\phi_{1}(z)-s_{n}(z) \equiv \frac{1}{2 \pi i} \int_{\gamma} \frac{\omega_{n}(z)\left[\phi_{1}(t)-R_{n}(t)\right] d t}{\omega_{n}(t)(t-z)}, \quad z \text { in } D_{0}^{\prime} .
$$

Each region $E_{j}$ disjoint from $D_{\rho}$ whose boundary $\Gamma_{\rho}^{j}$ consists wholly of one or more components of $\Gamma_{\rho}$ contains one or more points $b_{i}$, of which we choose a particular one, say $b_{j}$; the $E_{j}$ and hence the $b_{j}$ may be fewer than $\nu$ in number. The open set $D_{0}^{\prime}$ is bounded by $\Gamma_{\rho}$, namely $\sum_{j} \Gamma_{\rho}^{j}$, and $\phi_{1}(z)$ can be expressed for $z$ in $D_{0}^{\prime}$

$$
\begin{aligned}
\phi_{1}(z) & \equiv \sum_{j} \psi_{j}(z), \\
\psi_{j}(z) & \equiv \frac{1}{2 \pi i} \int_{\mathrm{r}_{\rho}^{j}} \frac{\phi_{1}(t) d t}{t-z},
\end{aligned}
$$

where $\psi_{j}(z)$ is analytic throughout the complement of $E_{j}$, and where $\psi_{j}(z)$ can be considered defined on $\Gamma_{\rho}^{\prime}$ by $(20)$, hence is of class $H(k, \alpha, p)$ there. For each $j$ and $N_{j}=1,2, \cdots$ a polynomial $P_{N_{j}}\left[1 /\left(z-b_{j}\right)\right]$ in $1 /\left(z-b_{j}\right)$ of degree $N_{j}$ exists such that we have

$$
\int_{\Gamma_{\rho}^{j}}\left|\psi_{j}(z)-P_{N_{j}}\left[1 /\left(z-b_{j}\right)\right]\right| p|d z| \leqq A / N_{j}^{(k+\alpha) p} ;
$$

in fact, when the configuration is transformed by the substitution $w=1 /\left(z-b_{j}\right)$ the image satisfies precisely the conditions of [4, Theorem 1$]$, since $\psi_{j}(z)$ belongs to the class $H(k, \alpha, p)$ on $\Gamma_{\rho}^{j}$, and the class is invariant under such a transformation. For each $n(=1,2,3, \cdots)$ we now choose $N_{j}$ as the $N_{n j}$ previously introduced in connection with (14); we have $\sum_{i} N_{n i}=n$, but $\sum_{j} N_{j}=\sum_{j} N_{n j} \leqq n$, because not all $b_{i}$ need occur among the $b_{j}$. The function $\sum_{j} P_{N_{j}}\left[1 /\left(z-b_{j}\right)\right]$ is a rational function $R_{n}(z)$ of degree $n$ whose poles 
(multiplicity considered) lie in the points $\left(\beta_{1}, \beta_{2}, \cdots, \beta_{n}\right)$. It is to be noted that by Cauchy's integral formula for $\psi_{j}(z)-P_{N_{j}}\left[1 /\left(z-b_{j}\right)\right]$ taken over $\Gamma_{\rho}^{j}$, the analogue of (22) holds when the integral is taken over $\Gamma_{\rho}^{\lambda}, \lambda \neq j$, with change of $A$ but without change of the integrand. Since the numbers $n / N_{n j}$ are bounded, we may by (20) sum the inequalities (22) and these analogues $\left.{ }^{4}\right)$ :

$$
\int_{\mathrm{r}_{\rho}}\left|\phi_{1}(z)-R_{n}(z)\right| p|d z| \leqq A_{1} / n^{(k+\alpha) p}
$$

We may replace $\gamma$ in (19) by $\Gamma_{\rho}$, whence by (15), (19), and (23)

$$
\left|\phi_{1}(z)-s_{n}(z)\right| \leqq A_{2} e^{-n \rho / \tau} / n^{k+\alpha},
$$

$z$ on $B$,

since we have $U(z) \equiv\left[\log |u(z)|-d_{0}\right] /\left(d_{1}-d_{0}\right), \tau=1 /\left(d_{1}-d_{0}\right)$. Inequality (11) follows from (24) if we set $f_{n}(z) \equiv s_{n}(z)+\phi_{2}(z) \equiv s_{n}(z)+f(z)-\phi_{1}(z)$. Inequality (12) follows from (24) as in [1] by the special properties of the series (14), and this completes the proof of Theorem 6 in the case $k \geqq 0$.

With $k<0$ we have as a consequence of Theorem 4 an inequality for the norm of $f(z)$ not merely on the images of the level loci $|z|=$ const when the interior (or exterior) of a component $\Gamma_{\rho}^{\prime}$ of $\Gamma_{\rho}$ is mapped onto the unit disc, but also an inequality for the norm of $f(z)$ and hence that of $\phi_{1}(z)$ on the level loci of $U(z)$ :

$$
\int_{\Gamma_{r}}\left|\phi_{1}(z)\right| p|d z| \leqq A(\rho-r)^{(k+\alpha) p}, \quad r_{0}<r<\rho .
$$

Equation (17) for $z$ on $B$ with $\gamma$ chosen as $\Gamma_{r}, r=r_{n}=\rho(1-1 / n)$, yields again (24) and (11). Inequality (12) can also be obtained easily [1] by the special properties of the series (14), so Theorem 6 is established.

The converse of Theorem 5 is false, but if the functions $f_{n}(z)$ and $f(z)$ are given continuous on $B$ and $f_{n}(z)$ analytic in $D$ satisfying (11) and (12), it can be shown [1] that $f(z)$ satisfying (11) exists analytic in $D_{\rho}$, continuous on $B$, and of class $H^{\prime}(k-1, \alpha, \infty)$ on $\Gamma_{\rho}$. This discrepancy of unity in the classes involved is inherent in the nature of the problem, as examples show. Nevertheless the weaker hypothesis of the classes $H^{\prime}(k, \alpha, p)$ in Theorem 6 represent a decrease in that discrepancy, and this is the primary interest here of Theorem 6.

(4) We note in passing the following theorem, whose proof has essentially been given above; an analogue for functions of class $H^{\prime}(k, \alpha, \infty)$ can replace [12] in the treatment given in [1], with material simplification:

Let $E$ be a bounded open set of the z-plane whose boundary $J$ consists of a finite number of mutually disjoint analytic Jordan curves $J_{j}, J=\sum J_{j}$. Let $\phi_{1}(z)$ be analytic on $E$, of class $H^{\prime}(k, \alpha, p)$ on $J, 0<\alpha<1, p \geqq 1$. In the extended plane let the set complementary to $E+J$ consist of the mutually disjoint regions $E_{1}, E_{2}, \cdots, E_{\lambda}$. Let each $E_{k}$ contain at least one of the points $b_{1}, b_{2}, \cdots, b_{\nu}$, let the sequence $\beta_{1}, \beta_{2}, \cdots$ consist only of points $b_{j}$, and if $N_{n j}$ denotes the number of times $b_{j}$ occurs in the set $\beta_{1}, \beta_{2}, \cdots, \beta_{n}$, let $n / N_{n j}$ be bounded for all $j$ and $n \geqq \nu$. Then there exist rational functions $R_{n}(z)$ of respective degrees $n$ whose poles lie in the points $\beta_{1}, \beta_{2}, \cdots, \beta_{n}$ such that (23) is valid with the integral taken over $J$ instead of $\Gamma_{\rho}$. 
In Theorem 6 we have omitted the classes $H(k, 1, p)$, but substitute classes involving suitable integrated continuity conditions can be used without difficulty; these classes were introduced into the theory of trigononometric approximation by Zygmund and into the study of analytic functions by Walsh and Russell [4]; for $p=\infty$ compare Walsh and Elliott [8]. If the function $f(z)$ is analytic in a one-sided neighborhood of an analytic Jordan curve $J$ and of class $H_{p}$ there, it belongs to class $Z^{\prime}(k, p), k \geqq 0$, on $J$ if for the boundary values $f(z)$ the $k$ th derivative $f^{(k)}(z) \equiv F(s)$ exists continuously on $J$ and satisfies

$$
\int_{J}|F(s+h)+F(s-h)-2 F(s)|^{p} d s \leqq A|h|^{p},
$$

where $A$ is independent of $h$. The function $f(z)$ analytic in a neighborhood $\rho<|z|<1$ of $\gamma:|z|=1$ is of class $Z^{\prime}(k, p), k<-1$, on $\gamma$ if we have in that neighborhood

$$
\int_{|z|=r}\left|f\left(r e^{i \theta}\right)\right|^{p} d s \leqq A(1-r)^{(k+1) p} .
$$

A function $f(z)$ analytic in a one-sided neighborhood of an analytic Jordan curve $J$ is said to be of class $Z^{\prime}(k, p)$ on $J, k<-1$, if its transform is of class $Z^{\prime}(k, p)$ on $\gamma$ when the interior (or exterior) of $J$ is mapped onto the interior of $\gamma$. There exists again a sequence of classes $\cdots Z^{\prime}(-2, p), Z^{\prime}(-1, p)$, $Z^{\prime}(0, p), Z^{\prime}(1, p), \cdots$; the derivative and integral of a function of a class have the properties mentioned in connection with the sequence $H^{\prime}(k, \alpha, p)$. Analogues of Theorems 1, 2, 3, and 4 can be proved. The method of proof already used shows that Theorem 6 can be enlarged by the statment that if $f(z)$ is analytic in $D_{\rho}$, continuous on $B$, and of class $Z^{\prime}(k, p)$ on $\Gamma_{p}$, then there exist functions $f_{n}(z)$ analytic in $D$ and continuous on $B$ such that (11) and (12) are valid with $\alpha=1$.

In Theorem 6 (including the enlargement) if $D$ lies interior to $C_{1}$, if the Jordan curves $B_{j}$ are analytic, and if $f(z)$ is analytic on and within each $B_{j}$, the functions $f_{n}(z)$ can be chosen analytic in $D$ and also on and within each $B_{j}$; compare [1, Theorem 6].

The topological requirements of Theorem 5 can be greatly broadened [13] without essential change of the conclusion. A corresponding broadening of Theorem 6 can be achieved by use of Theorem 6 together with the remarks just made, precisely as in [13]:

Theorem 7. Let $B_{1}, B_{2}, \cdots, B_{\mu}, C_{1}, C_{2}, \cdots, C_{\nu}$ be a set of mutually disjoint analytic Jordan curves; we denote by $B$ the sum of the $B_{j}$ and by $C$ the sum of the $C_{j}$. Let $D$ be a bounded open set whose boundary is all of $C$ plus a (not necessarily proper) subset of the $B_{j}$, and indeed let each maximal (i.e., contained in no larger) subregion of $D$ be bounded by a nonempty subset of the $B_{j}$ plus a nonempty subset of $C$. Let $C$ not separate $D$. Let $E$ be a closed set disjoint from $D$ 
whose boundary is $B$; but $E$ need not be the closure of an open set. Let the set $D+E$ be connected.

Let $U(z)$ be the unique function harmonic in $D$, continuous in the closure of $D$, equal to zero on $B$ and unity on $C$. Let $\Gamma_{\sigma}, 0<\sigma<1$, denote generically the locus $U(z)=\sigma$ in $D$, and let $D_{\sigma}$ denote the set $0<U(z)<\sigma$ in $D$.

Let the function $f(z)$ be analytic in the interior points of $E$, continuous on $E$, and also analytic throughout $D_{\rho}$, where $\Gamma_{\rho}$ has no multiple points; thus $f(z)$ is analytic in the interior points of $E+D_{\rho}$. Let $f(z)$ on $\Gamma_{\rho}$ be of class $H^{\prime}(k, \alpha, p)$ for $0<\alpha<1$ or $Z^{\prime}(k, p)$ for $\alpha=1$.

Then there exist functions $f_{n}(z)$ analytic in the interior points of $D+E$ and continuous on E, satisfying the inequalities (11) and (12).

Here, as in Theorems 5 and 6, the number $\tau$ in the inequalities (11) and (12) may be replaced by unity. In fact, the inequalities may be replaced by others involving a continuous parameter instead of the sequence $n=1,2, \cdots$; compare $[1, \S 8]$.

\section{REFERENCES}

1. J. L. Walsh, On approximation by bounded analytic functions, Trans. Amer. Math. Soc. vol. 87 (1958) pp. 467-484.

2. G. H. Hardy and J. E. Littlewood, A convergence criterion for Fourier series, Math. Z. vol. 28 (1928) pp. 612-634.

3. E. S. Quade, Trigonometric approximation in the mean, Duke Math. J. vol. 3 (1937) pp. 529-543.

4. J. L. Walsh and H. G. Russell, Integrated continuity conditions and degree of approximation by polynomials or by bounded analytic functions, Trans. Amer. Math. Soc. vol. 92 (1959) pp. 355-370.

5. J. L. Walsh, Note on approximation by bounded analytic functions (Problem $\alpha$ ), Math. Z. vol. 72 (1959) pp. 47-52.

6. - Note on invariance of degree of polynomial and trigonometric approximation under change of independent variable, Proc. Nat. Acad. Sci. U.S.A. vol. 45 (1959) pp. 1528-1533.

7. - Sur l'approximation par fonctions rationnelles et par fonctions holomorphes bornées, Ann. Mat. Pura Appl. (4) vol. 39 (1955) pp. 269-277.

8. J. L. Walsh and H. M. Elliott, Polynomial approximation to harmonic and analytic functions: generalized continuity conditions, Trans. Amer. Math. Soc. vol. 68 (1950) pp. 183-203.

9. J. L. Walsh, On the conformal mapping of multiply connected regions, Trans. Amer. Math. Soc. vol. 82 (1956) pp. 128-146.

10. - Interpolation and approximation, Amer. Math. Soc. Colloquium Publications, vol. $20,1935$.

11. J. L. Walsh and W. E. Sewell, Note on degree of trigonometric and polynomial approximation to an analytic function, in the sense of least pth powers, Bull. Amer. Math. Soc. vol. 46 (1940) pp. 312-319.

12. J. L. Walsh, Note on degree of approximation to analytic functions by rational functions with preassigned poles, Proc. Nat. Acad. Sci. U.S.A. vol. 42 (1956) pp. 927-930.

13. - Approximation by bounded analytic functions: general configurations, Proc. Amer. Math. Soc. vol. 10 (1959) pp. 280-285.

HARVARD UNIVERSITY, Cambridge, Massachusetts 